CREAT. MATH. INFORM.

Volume 25 (2016), No. 2,

Pages 135 - 140
Online version at https : //creative-mathematics. cunbm . utcluj. ro/

Print Edition: ISSN 1584 - 286X; Online Edition: ISSN 1843 - 441X

DOI: https://doi.org/10.37193/CMI.2016.02.03

\title{
An empirical study of the convergence area and convergence speed of Agarwal et al. fixed point iteration procedure
}

\author{
GHEORGHE ARDELEAN and LASZLO BALOG
}

ABSTRACT. We present an empirical study of the convergence area and speed of Agarwal et al. fixed point iterative procedure in the particular case of the Newton's method associated to the complex polynomials $p_{3}(z)=$ $z^{3}-1$ and $p_{8}(z)=z^{8}-1$. In order to obtain an analytical expression for the experimental data related to the mean number of iterations (MNI) and convergence area index (CAI), we use regression analysis and find some linear and nonlinear bi-variable models with good correlation coefficients.

\section{INTRODUCTION}

It is well-nkown that the problem of solving the equation $f(x)=0$ can be equivalently transformed into a fixed point problem $T(x)=x$, where $T$ is some operator. Then, applying different iterations procedures for fixed points approximation, one can approximate the solution of the given equation.

Let $(X, d)$ be a complete metric space and $T: X \rightarrow X$ a selfmap on $X$. The set $F_{T}=$ $\{x \in X / T(x)=x\}$ is the set of all fixed points of $T$. The operator $T$ is usually called iteration function.

Many iterative processes for the approximation of fixed points have been described in the literature [3-6]. In the following, assume that each iteration process starts from any initial point $x_{0} \in X$.

\section{ITERATION PROCEDURES FOR FIXED POINTS APPROXIMATION}

The Picard iteration [7] (E. Picard, 1890), was defined as:

$$
x_{n+1}=T\left(x_{n}\right), \quad n=0,1,2, \cdots
$$

As an example, if the operator $T$ is defined as

$$
T(x)=x-\frac{f(x)}{f^{\prime}(x)}
$$

then the Picard iteration becomes

$$
x_{n+1}=T\left(x_{n}\right)=x_{n}-\frac{f\left(x_{n}\right)}{f^{\prime}\left(x_{n}\right)}, n=0,1,2, \cdots
$$

which is the well-known Newton method for solving the nonlinear equation $f(x)=0$.

The Agarwal et al. iteration [1] (Agarwal et al., 2007), was defined as the following two-step process:

$$
\begin{gathered}
x_{n+1}=\left(1-\alpha_{n}\right) T\left(x_{n}\right)+\alpha_{n} T\left(y_{n}\right), \\
y_{n}=\left(1-\beta_{n}\right) x_{n}+\beta_{n} T\left(x_{n}\right), \quad n=0,1,2, \cdots
\end{gathered}
$$

Received: 05.04.2016. In revised form: 04.05.2016. Accepted: 30.06.2016

2010 Mathematics Subject Classification. 47H10, 65H05.

Key words and phrases. Fixed point, Agarwal et al. iteration, convergence, number of iterations.

Corresponding author: Gheorghe Ardelean; ardelean_g@cunbm.utcluj.ro 
where $\alpha_{n} \in[0,1]$ and $\beta_{n} \in[0,1]$ for all $n \in \mathbb{N}$.

\section{NUMERICAL RESULTS}

Newton's method for finding the roots of a complex polynomial $p(z)$ is given by the formula

$$
\begin{gathered}
z_{n+1}=N\left(z_{n}\right), \quad n=0,1,2, \cdots \\
N(z)=z-\frac{p(z)}{p^{\prime}(z)}
\end{gathered}
$$

where $p^{\prime}(z)$ is the first derivative of $p$ and $z_{0} \in \mathbb{C}$ is a starting point.

If the sequence $\left\{z_{n}\right\}_{n=0}^{\infty}$ (the orbit of the point $z_{0}$ ) converges to a root $z^{*}$ of the polynomial $p$ then we say that $z_{0}$ is attracted by $z^{*}$. The attraction basin of the root $z^{*}$ of the polynomial $p$ is the set of all starting points $z_{0}$ which are attracted by $z^{*}$. We consider that the iteration method converges if the residual is less than $10^{-5}$ in a maximum of 13 iterations.

In [2] we present a comparative study of some iterative methods for solving nonlinear equations by using the basins of attraction.

To empirical evaluate the behaviors of Agarwal et al. iteration process, we considered the complex polynomials

$$
P_{3}(z)=z^{3}-1 \text { and } P_{8}(z)=z^{8}-1 \text {, respectively }
$$

In this case, the fixed point problems are

$$
z=\frac{2 z^{3}+1}{3 z^{2}}, \text { and } z=\frac{7 z^{8}+1}{8 z^{7}}, \text { respectively, } z \in \mathbb{C}
$$

so

$$
N_{3}(z)=\frac{2 z^{3}+1}{3 z^{2}} \text { and } N_{8}(z)=\frac{7 z^{8}+1}{8 z^{7}}, z \in \mathbb{C} .
$$

We take the rectangle in the complex plane $D=[-1.5,1.5] \times[-1.5,1.5]$, and then divide the rectangle into $250 \times 250$ grids. We apply the simplified Agarwal et al. iteration process for the Newton operators defined in (3.7)

$$
\begin{gathered}
z_{n+1}=(1-\alpha) N\left(z_{n}\right)+\alpha N\left(y_{n}\right), \\
y_{n}=(1-\beta) z_{n}+\beta N\left(z_{n}\right), \quad n=0,1,2, \cdots
\end{gathered}
$$

starting at each grid point.

We compute the mean number if iterations denoted $M N I$, and converging area index denoted $C A I$ (i.e. $C A I=\frac{\text { Number of converging points }}{\text { Total number of grid points }}$ ) for each pair

$$
(\alpha, \beta) \in\{0.1,0.2,0.3,0.4,0.5,0.6,0.7,0.8,0.9\} \times\{0.1,0.2,0.3,0.4,0.5,0.6,0.7,0.8,0.9\} .
$$

The results are presented in Tables 1-4.

All numerical results were computed by using Matlab R2012a [8] mathematical software package from MathWorks. 
An empirical study of the convergence area and convergence speed of Agarwal et al. fixed point iteration procedure 137

Table 1. MNI for Agarwal et al. iteration process and the polynomial

$$
P_{3}(z)=z^{3}-1
$$

\begin{tabular}{cccccccccc}
\hline$\alpha \backslash \beta$ & 0.1 & 0.2 & 0.3 & 0.4 & 0.5 & 0.6 & 0.7 & 0.8 & 0.9 \\
\hline 0.1 & 5.6722 & 5.6220 & 5.6063 & 5.5165 & 5.4362 & 5.3166 & 5.2516 & 5.1763 & 5.1421 \\
0.2 & 5.6167 & 5.5668 & 5.3985 & 5.2566 & 5.1722 & 5.0400 & 4.9628 & 4.8334 & 4.7946 \\
0.3 & 5.6158 & 5.4081 & 5.0812 & 5.0812 & 4.9600 & 4.8011 & 4.6995 & 4.5931 & 4.4369 \\
0.4 & 5.5542 & 5.2799 & 5.0718 & 4.9022 & 4.7770 & 4.6212 & 4.5068 & 4.3461 & 4.2674 \\
0.5 & 5.4872 & 5.2002 & 4.9816 & 4.8047 & 4.6734 & 4.4938 & 4.3388 & 4.1257 & 3.9904 \\
0.6 & 5.4032 & 5.1051 & 4.8763 & 4.7115 & 4.5225 & 4.3384 & 4.2610 & 3.756 & 6.424 \\
0.7 & 5.3452 & 5.0535 & 4.719 & 4.6388 & 4.4371 & 4.2807 & 4.1631 & 4.0375 & 3.8080 \\
0.8 & 5.3035 & 4.9707 & 4.7309 & 4.5623 & 4.3974 & 4.2313 & 4.0980 & 3.9745 & 3.7447 \\
0.9 & 5.2412 & 4.9681 & 4.7135 & 4.5287 & 4.3106 & 4.2131 & 4.0859 & 3.8706 & 3.7230 \\
\hline
\end{tabular}

Table 2. CAI for Agarwal et al. iteration process and the polynomial

$$
P_{3}(z)=z^{3}-1
$$

\begin{tabular}{cccccccccc}
\hline$\alpha \backslash \beta$ & 0.1 & 0.2 & 0.3 & 0.4 & 0.5 & 0.6 & 0.7 & 0.8 & 0.9 \\
\hline 0.1 & 0.9419 & 0.9458 & 0.9658 & 0.9685 & 0.9758 & 0.9750 & 0.9781 & 0.9815 & 0.9877 \\
0.2 & 0.9408 & 0.9673 & 0.9735 & 0.9754 & 0.9846 & 0.9812 & 0.9919 & 0.9923 & 0.9938 \\
0.3 & 0.9546 & 0.9685 & 0.9685 & 0.9847 & 0.9896 & 0.9858 & 0.9904 & 0.9912 & 0.9927 \\
0.4 & 0.9608 & 0.9696 & 0.9796 & 0.983 & 0.9912 & 0.9896 & 0.9923 & 0.9919 & 0.9935 \\
0.5 & 0.9731 & 0.9797 & 0.9804 & 0.9885 & 0.9935 & 0.9942 & 0.9931 & 0.9942 & 0.9958 \\
0.6 & 0.9715 & 0.9800 & 0.9885 & 0.9915 & 0.9912 & 0.9908 & 0.9958 & 0.9942 & 0.9965 \\
0.7 & 0.9735 & 0.9846 & 0.9873 & 0.9919 & 0.9938 & 0.9942 & 0.9946 & 0.9958 & 0.9950 \\
0.8 & 0.9792 & 0.9831 & 0.9915 & 0.9908 & 0.9946 & 0.9938 & 0.9963 & 0.9958 & 0.9969 \\
0.9 & 0.9789 & 0.9981 & 0.9904 & 0.9912 & 0.9938 & 0.9958 & 0.9981 & 0.9954 & 0.9981 \\
\hline
\end{tabular}

Figures 1-4 present the analytical expression for MNI and CAI, respectively, depending on $\alpha$ and $\beta$, generated by using the TableCurve3D v4.0 [9] program of SYSTAT Software Inc. for surface fitting.

Surface fitting refers to finding an appropriate mathematical model that expresses the relationship between a dependent variable $\mathrm{z}$ and two independent variables $\alpha$ and $\beta$, and estimating the values of its parameters using nonlinear regression.

In Figure 1

$$
z=\operatorname{MNI}(\alpha, \beta)=a+b \alpha+c \beta
$$

with $a=6.144234, b=-1.2116667$ and $c=-1.5233074$, the mean number of iterations $\operatorname{MNI}(\alpha, \beta)$ is approximated as a linear function on $\alpha$ and $\beta$ (by using Least Ssquares Mmethod for surface fitting). The value of the accuracy indicator $r^{2}=0.93160209$ shows that the approximation is a very good one.

In Figure 2

$$
z=C A I(\alpha, \beta)=a+b \alpha+c / \beta+d \alpha^{2}+e / \beta^{2}+f \alpha / \beta
$$

with $a=0.98837987, b=0.048021787, c=-0.0093104925, d=-0.035891053, e=$ 0.00032273351 and $f=0.0047416604$, the Convergence Area Index $C A I(\alpha, \beta)$ is approximated as a rational function on $\alpha$ and $\beta$. The value of the accuracy indicator $r^{2}=0.93304197$ shows that the approximation is very good too. 
Table 3. MNI for Agarwal et al. iteration process and the polynomial

$$
P_{8}(z)=z^{8}-1
$$

\begin{tabular}{cccccccccc}
\hline$\alpha \backslash \beta$ & 0.1 & 0.2 & 0.3 & 0.4 & 0.5 & 0.6 & 0.7 & 0.8 & 0.9 \\
\hline 0.1 & 5.6722 & 5.6220 & 5.6063 & 5.5165 & 5.4362 & 5.3166 & 5.2516 & 5.1763 & 5.1421 \\
0.2 & 5.6167 & 5.5668 & 5.3985 & 5.2566 & 5.1722 & 5.0400 & 4.9628 & 4.8334 & 4.7946 \\
0.3 & 5.6158 & 5.4081 & 5.0812 & 5.0812 & 4.9600 & 4.8011 & 4.6995 & 4.5931 & 4.4369 \\
0.4 & 5.5542 & 5.2799 & 5.0718 & 4.9022 & 4.7770 & 4.6212 & 4.5068 & 4.3461 & 4.2674 \\
0.5 & 5.4872 & 5.2002 & 4.9816 & 4.8047 & 4.6734 & 4.4938 & 4.3388 & 4.1257 & 3.9904 \\
0.6 & 5.4032 & 5.1051 & 4.8763 & 4.7115 & 4.5225 & 4.3384 & 4.2610 & 3.756 & 6.424 \\
0.7 & 5.3452 & 5.0535 & 4.719 & 4.6388 & 4.4371 & 4.2807 & 4.1631 & 4.0375 & 3.8080 \\
0.8 & 5.3035 & 4.9707 & 4.7309 & 4.5623 & 4.3974 & 4.2313 & 4.0980 & 3.9745 & 3.7447 \\
0.9 & 5.2412 & 4.9681 & 4.7135 & 4.5287 & 4.3106 & 4.2131 & 4.0859 & 3.8706 & 3.7230 \\
\hline
\end{tabular}

Table 4. CAI for Agarwal et al. iteration process and the polynomial

$$
P_{8}(z)=z^{8}-1
$$

\begin{tabular}{cccccccccc}
\hline$\alpha \backslash \beta$ & 0.1 & 0.2 & 0.3 & 0.4 & 0.5 & 0.6 & 0.7 & 0.8 & 0.9 \\
\hline 0.1 & 0.9419 & 0.9458 & 0.9658 & 0.9685 & 0.9758 & 0.9750 & 0.9781 & 0.9815 & 0.9877 \\
0.2 & 0.9408 & 0.9673 & 0.9735 & 0.9754 & 0.9846 & 0.9812 & 0.9919 & 0.9923 & 0.9938 \\
0.3 & 0.9546 & 0.9685 & 0.9685 & 0.9847 & 0.9896 & 0.9858 & 0.9904 & 0.9912 & 0.9927 \\
0.4 & 0.9608 & 0.9696 & 0.9796 & 0.983 & 0.9912 & 0.9896 & 0.9923 & 0.9919 & 0.9935 \\
0.5 & 0.9731 & 0.9797 & 0.9804 & 0.9885 & 0.9935 & 0.9942 & 0.9931 & 0.9942 & 0.9958 \\
0.6 & 0.9715 & 0.9800 & 0.9885 & 0.9915 & 0.9912 & 0.9908 & 0.9958 & 0.9942 & 0.9965 \\
0.7 & 0.9735 & 0.9846 & 0.9873 & 0.9919 & 0.9938 & 0.9942 & 0.9946 & 0.9958 & 0.9950 \\
0.8 & 0.9792 & 0.9831 & 0.9915 & 0.9908 & 0.9946 & 0.9938 & 0.9963 & 0.9958 & 0.9969 \\
0.9 & 0.9789 & 0.9981 & 0.9904 & 0.9912 & 0.9938 & 0.9958 & 0.9981 & 0.9954 & 0.9981 \\
\hline
\end{tabular}

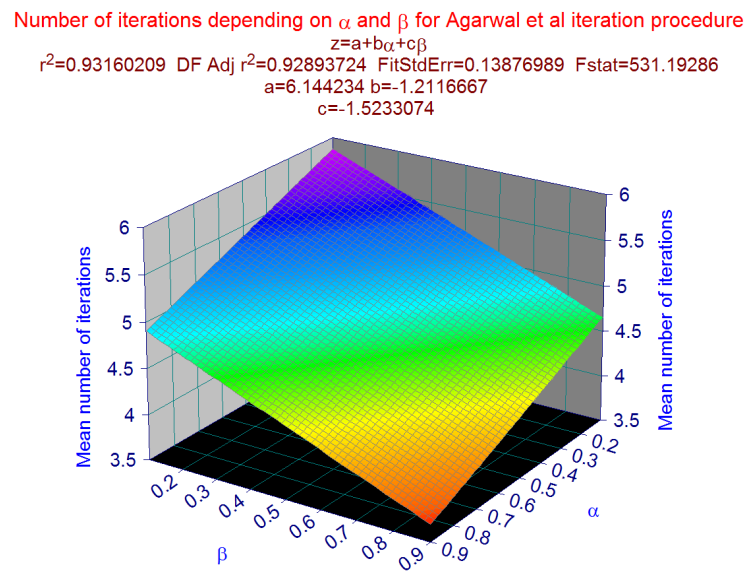

Figure 1. Mean number of iterations (MNI) for the polynomial $P_{3}(z)$ 

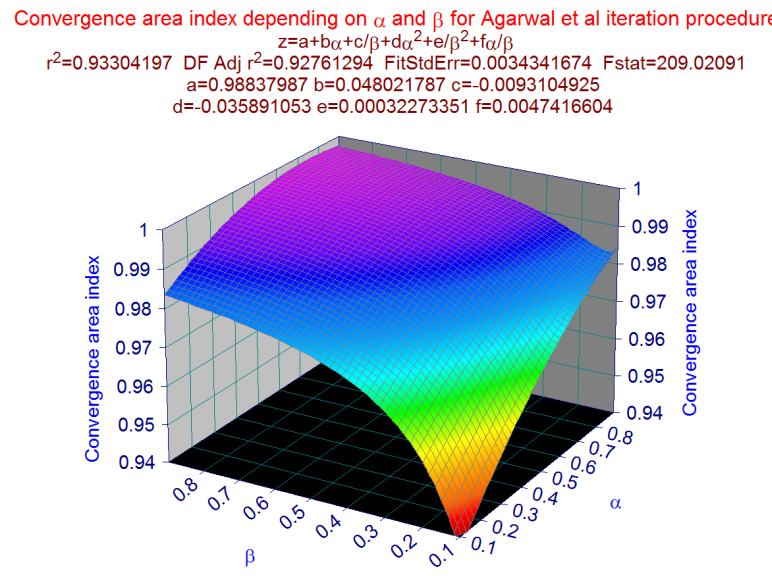

Figure 2. Convergence area index (CAI) for the polynomial $P_{3}(z)$

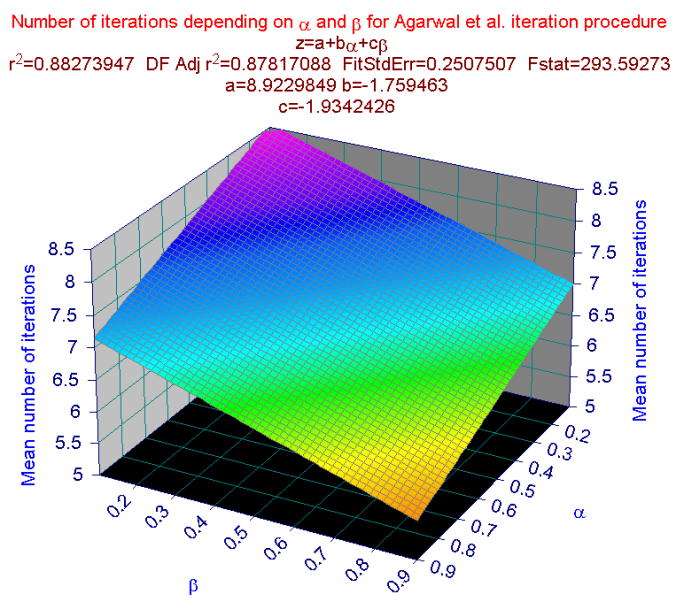

Figure 3. Mean number of iterations (MNI) for the polynomial $P_{8}(z)$

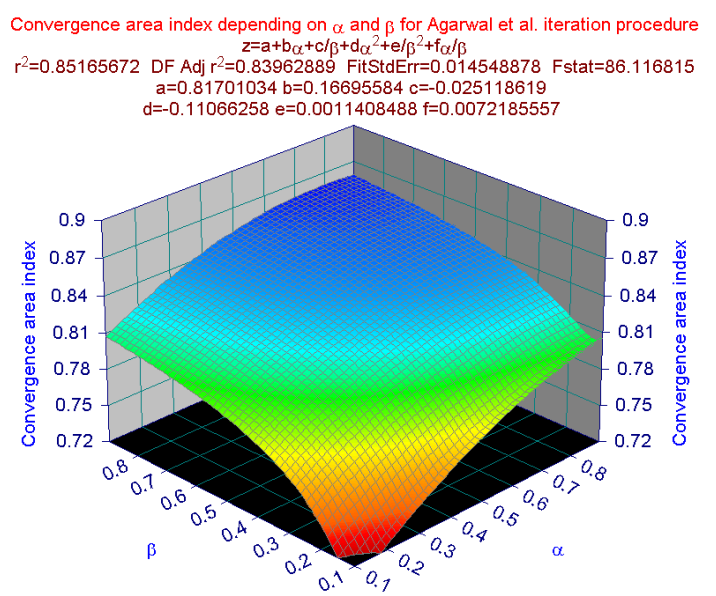

Figure 4. Convergence area index (CAI) for the polynomial $P_{8}(z)$ 


\section{CONCLUSIONS}

The numerical results in Table 1 and Table 3 and the graphs in Figure 1 and Figure 3 shows that, for fixed point problems (3.7), the Mean Number of Iterations is decreasing while the values of $\alpha$ and $\beta$ are increasing (the minimum number of iterations is obtained for $\alpha=0.9$ and $\beta=0.9$ ).

The numerical results in Table 2 and Table 4 and the graphs in Figure 2 and Figure 4 shows that, for fixed point problems (3.7), the Convergence Area Index is increasing while the values of $\alpha$ and $\beta$ are increasing (the maximum Convergence Area Index is obtained for $\alpha=0.9$ and $\beta=0.9)$.

Acknowledgement. The authors would like to thank the referees for their useful comments and constructive suggestions which substantially improved the quality of this paper.

\section{REFERENCES}

[1] Agarwal, R. P., O'Regan, D. and Sahu, D. R., Iterative construction of fixed points of nearly asymptotically nonexpansive mappings, J. Nonlinear Convex Anal., 8 (2007), No. 1, 61-79

[2] Ardelean, G., A comparison between iterative methods by using the basins of attraction, Appl. Math. Comput., 218 (2011), 88-95

[3] Berinde V., Iterative Approximation of Fixed Points, 2nd edn. Springer, Heidelberg, 2007

[4] Berinde, V., On the convergence of the Ishikawa iteration in the class of quasi-contractive operators, Acta Math. Univ. Comenian. (N.S.), 73 (2004), No. 1, 119-126

[5] Chugh, R. and Kumar, S., On the Stability and Strong Convergence for Jungck-Agarwal et al. Iteration Procedure, Int. J. Comput. Appl., (0975 - 8887) 64, No. 7, 2013

[6] Gdawiec, K., Kotarski, W. and Lisowska, A., Polynomiography with Non-standard Iterations, WSCG 2014 Poster Papers Proceedings, 21-26

[7] Picard, E., Mémoire sur la théorie des équations aux dérivées partielles et la méthose des approximations successives, J. Math. Pures Appl., 6 (1890), No. 4, 145-210

[8] *** www.mathworks.com

[9] *** www.systatsoftware.com

TECHNICAL UNIVERSITY OF CLUJ-NAPOCA

North University CENTER AT BAIA MARE

DEPARTMENT OF MATHEMATICS AND COMPUTER SCIENCE

VICTORIEI 76, 430122 BAIA MARE, ROMANIA

Email address: ardelean_g@cunbm.utcluj.ro

Email address: balog_58@yahoo.com 